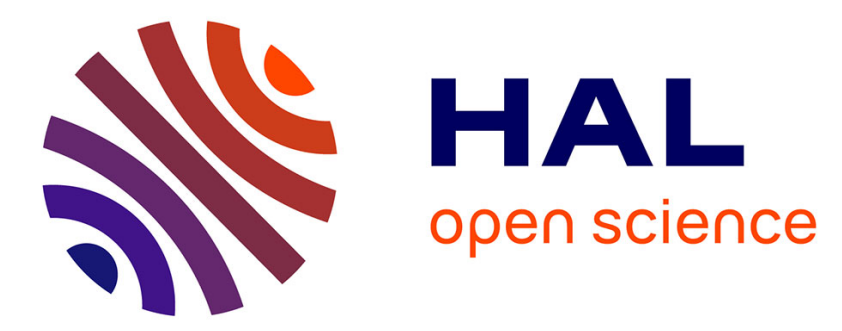

\title{
Développement professionnel infirmier en promotion de la santé
}

\author{
Anne-France Hardy, Jérôme Eneau, Marc Nagels
}

\section{To cite this version:}

Anne-France Hardy, Jérôme Eneau, Marc Nagels. Développement professionnel infirmier en promotion de la santé. Recherche en soins infirmiers, 2014, 118 (118), pp.74-83. 10.3917/rsi.118.0075 . hal01162836

\section{HAL Id: hal-01162836 \\ https://hal.science/hal-01162836}

Submitted on 13 Mar 2018

HAL is a multi-disciplinary open access archive for the deposit and dissemination of scientific research documents, whether they are published or not. The documents may come from teaching and research institutions in France or abroad, or from public or private research centers.
L'archive ouverte pluridisciplinaire HAL, est destinée au dépôt et à la diffusion de documents scientifiques de niveau recherche, publiés ou non, émanant des établissements d'enseignement et de recherche français ou étrangers, des laboratoires publics ou privés. 


\title{
Développement professionnel infirmier en promotion de la santé
}

\author{
Professional development of nurses in promoting health
}

\section{Anne.France HARDY}

Infirmière, Inspection académique 35, Master, Education, Apprentissage, Didactique, Université Rennes2.

Sous la direction de :

Jérôme Eneau

$\mathrm{PhD}$ en Andragogie (Université de Montréal), Docteur en Sciences de l'Education, maître de conférences HDR, Université européenne de Bretagne- Département des Sciences de l'Education (CREAD, EA 3875)

\section{Marc Nagels}

Docteur en Sciences de l'Education, Université européenne de Bretagne- Rennes2 Département des Sciences de l'Education (CREAD, EA 3875)

Place du Recteur Le Moal. 35043 Rennes Cedex

\section{Résumé}

$\overline{\text { Cette recherche se propose d'éclairer le développement professionnel du personnel infirmier }}$ éducateur de santé en milieu scolaire. Les missions d'éducation et de promotion de la santé relèvent du savoir spécifique infirmier et correspondent en dehors du champ habituel de la prescription aux pratiques relevant $\mathrm{du}$ rôle propre. L'hypothèse d'un lien entre rôle spécifiquement infirmier et développement professionnel guide ce travail. L'étude compare $(\mathrm{N}=30)$ approches professionnelles d'infirmières d'établissement d'enseignement en poste sur le département d' "Ille et Vilaine ». L'expérience des acteurs est interrogée pour tenter de dégager les éléments de ce développement professionnel autour de trois indicateurs clés : la posture, les dynamiques identitaires et la dimension collective de l'activité. L'enquête de type qualitative emprunte les outils d'une méthode hybride qui recourt à l'analyse parallèle de contenus recueillis sous deux modalités différentes: l'entretien compréhensif et l'écrit réflexif.

Mots clés : Développement professionnel, rôle propre infirmier, éducation et promotion de la santé

\begin{abstract}
This research aims at helping the nursing staff and it will help them to develop the skills to use among the school community. On top of her ordinary skills and beyond simple prescriptions, a nurse also has to teach and promote health. It is believed that there is a link between the professional and the personal development. This research deals with the different approaches of $(\mathrm{N}=30)$ nurses working within different schools in "Ille et Vilaine". Nurses 'experiences have been questioned to identify how this professional development centered on three issues: awareness; identity process and what group they belong to. This qualitive research lies on varied tools that use a compared analysis based on two different types: an oral interview and a pondering written part.
\end{abstract}

Keywords: professional development/nurse skills/education/promoting health

Adresse de correspondance :

Anne.France Hardy: afrhardy@gmail.com 


\section{Introduction}

Cette réflexion questionne les compétences de l'éducateur en santé ancrées dans le rôle propre infirmier et la démarche de promotion de la santé. Reconnu depuis 1978, par les instances politiques, les pratiques d'éducation à la santé relevant de ce rôle s'adressent à la personne et au groupe.

Enracinée dans la promotion de la vie (Collière, 1982) [1], la professionnalisation engagée depuis 1960 et concomitante à l'expansion des savoirs médicaux, a surtout instrumenté les pratiques professionnelles autour des soins se référant au "cure ». Le service adressé à la personne, relatif au " prendre soin » qu'identifie le concept du " care » tend à s'effacer vis-àvis de celui qui est rendu à l'institution (Collière, 1982) [1] ; (Piguet, 2008) [2]; (RothierBautzer, 2012) [3].

Depuis la Charte d'Ottawa, l'évolution des pratiques soignantes correspond à un enjeu de santé publique et le corps professionnel infirmier représente un levier pour la promotion et l'amélioration de la santé des populations (Pépin, Kérouac, Ducharme, 2010) [4].

Prolongeant le concept d'éducation à la santé, la profession de soignant en santé scolaire s'inscrit dans la continuité des orientations hygiénistes mises en place après la guerre. Son objectif est celui de la prévention et de la promotion de modes de vie sains. Axée sur la compréhension des attitudes et des comportements sanitaires des populations, la promotion de la santé stimule le développement d'approches professionnelles dans le champ de la prévention. Relayant la transmission de connaissances biomédicales, cette pratique issue des résultats épidémiologiques et mise en place au nom du principe de précaution vise à travers la réduction des risques la modification des comportements individuels (Lecorps Paturet, 1999) [5]; (Descarpentries, 2008) [6].

L'analyse tente d'expliciter l'émergence du rôle spécifique infirmier ancré dans les pratiques dites du « care » de prévention et d'éducation à la santé en lien aux définitions de l'OMS et à la perspective holiste qu'elle préconise. Elle propose une contribution à la compréhension du développement professionnel en particulier centré sur l'appropriation du rôle spécifiquement infirmier dans le champ des pratiques de santé, (Piguet, 2008) [2].

Se référant à la fois au champ de la santé et de l'éducation, la posture professionnelle du soignant-éducateur n'est pas sans ambiguïté dans la lisibilité des missions qui lui sont assignées (Thomann, Gabel, Senéterre, 1998) [7]. Le changement radical des pratiques professionnelles acquises en formation initiale induit la définition d'une nouvelle professionnalité articulée autour de compétences spécifiques (Berger, Mabrouck, Courty. 2009) [8]. Cette posture invite le soignant éducateur à reconfigurer ses pratiques lui offrant un champ d'action nouveau. Il apprend en situation et son expertise peut lui permettre d'adapter son savoir-faire aux caractéristiques de son insertion professionnelle en milieu scolaire.

Ce rôle spécifique questionne la nature du savoir infirmier mobilisé dans la mise en œuvre des pratiques de promotion de la santé. Son déploiement dit « autonome » dépend du contexte, mais l'absence de prescription médicale dans le milieu scolaire a présenté ici le cadre d'une analyse pertinente, les différentes missions offrant aux professionnels la possibilité d'organiser leur activité.

La recherche tente de rendre compte des activités développées dans la reconfiguration de cette posture professionnelle. Comprendre la spécificité de ce rôle peut se référer à ce que l'infirmier-e se sent apte à mettre en place selon son sentiment d'efficacité (Nagels, 2013) [9] et le développement de son pouvoir d'action (Piguet, 2008) [2].

L'analyse de l'expérience dans ce cadre d'étude instruit les recherches se référant à la formation et sert les enjeux relatifs à la professionnalisation en lien aux nécessités et préoccupations dont font l'objet actuellement l'évolution des études infirmières et la mutation du système de santé (Rothier-Bautzer, 2012) [3]. 
L'étude croise les questions éducatives relatives aux soins et se situe au carrefour de deux champs disciplinaires aux enjeux épistémologiques communs : Les sciences de l'éducation et la santé publique (Descarpentries, 2006) [10]; (Dominicé, Jacquemet, 2009) [11]. Dans le cadre d'une réflexion plus générale, la démarche offre la tentative d'apporter une contribution à l'émergence de la recherche en soins infirmiers.

\section{Déloppement professionnel : Ancrages théoriques}

L'enquête s'enracine dans le double ancrage épistémologique du constructivisme et du socioconstructivisme. La perspective constructiviste d'ordre phénoménologique correspond à l'étude d'un sujet singulier qui se construit dans son activité (Le Moigne, 2007) [12]; (Wittorski, 2007) [13]. Sous un angle vygotskien, la construction et le développement professionnel sont perçus comme un fait culturellement médiatisé (Rabardel, 2005) [14].

Le cadre théorique de la professionnalisation questionne le rôle propre à l'aune du développement professionnel. Analysé dans ses dimensions développementales et professionalisantes (Wittorski, 2007) [13] il l'explore dans l'espace spécifique de la promotion de la santé. Les indicateurs conceptuels destinés à en rendre compte interrogent la posture (Lameul, 2006) [15], la dimension collective de l'activité et les dynamiques identitaires à l'œuvre dans ce champ d'exercice.

Reconnu officiellement comme la parcelle d'un territoire institutionnel et symbolique, le rôle propre accompagne le processus de professionnalisation infirmier. Construit selon les contextes et les époques en référence aux définitions proposées par l'organisation mondiale de la santé (OMS), il suit l'évolution des représentations du concept de santé. Pourtant objet de décrets successifs, ce rôle associe non sans ambiguïtés, le positionnement, le raisonnement infirmier et les notions vagues de " pratiques autonomes ». Les éléments théoriques mobilisés tentent d'expliciter le développement et l'apprentissage infirmier dans les activités relevant de son savoir spécifique, enjeu essentiel de la professionnalisation, de son autonomie et de sa reconnaissance. Assortis d'actes de formalisation repérés et codifiés, ces savoirs ont une forte dimension sociale et identitaire pour affirmer la spécificité des activités du groupe d'appartenance (Wittorski, Ardouin 2012) [16].

Les connaissances se référant aux caractéristiques de la professionnalité infirmière suscitent peu de recherche prenant pour objet les pratiques professionnelles dans le champ de la prévention et de la promotion de la santé (Osiek-Parisod, 1994) [17]; (Batt, 1999) [18] ; (Berger, 2009) [8]. Ces infirmier-ères arrivent en contexte scolaire avec une identité professionnelle acquise en institut de formation et une conception de soin centrée sur le modèle curatif (Jourdan, 2004) [19]. Placé dans un contexte où domine la complexité des tâches discrétionnaires inhérentes aux activités de service, le soignant éducateur appréhende les problématiques de vie aux prises à une réalité sociale (Mayen, 2007) [20], dans un rôle professionnel spécifique associant à la fois des missions éducatives et de soin (Berger, Mabrouck, Courty, 2009) [8].

\section{Méthodologie}

Inscrite dans l'axe d'une approche qualitative de type empirico inductive, la méthode d'analyse emprunte les outils de "la grounded theory» issue de l'interactionnisme symbolique conceptualisée par Glaser et Strauss (Kaufmann, 1996) [21].

La perspective compréhensive de cette démarche d'analyse croise deux outils, l'entretien compréhensif (Kaufman, 1996) [21] et le récit d'expérience qu'offre le contenu de l'écrit réflexif (Cifali, André, 2007) [22]. L'ajout d'une approche narrative sollicitant sous un autre 
angle les dimensions praxéologiques, implicites et subjectives, a complété les méthodes classiques de l'entretien. L'enjeu de ce choix a été de susciter la démarche réflexive définie par Schön (1994) associant prise de conscience et conceptualisation.

La méthode d'écriture ayant par ailleurs l'avantage de diminuer l'effet des biais interprétatifs et des «perturbations » au sens de Devereux (1980), a postulé qu'une production personnelle se référant au vécu professionnel, puisse stimuler une autoréflexion. Cette dernière initiant le développement professionnel propre au sujet qui se questionne et ne fait pas que répondre à une prescription (Vinatier, 2012) [23]. Cette méthodologie ayant pour objectif d'atteindre des dimensions plus implicites de l'identité vécue (Wittorski, 2007) [13] et les formes opaques de l'expérience singulière nécessitant parfois décalage et distanciation (Dubet, 2000) [24].

Ces deux modalités de recueil ont été structurées autour de dix mots clés ${ }^{1}$ sélectionnés à l'issue d'une phase exploratoire. L'étude des entretiens compréhensifs d'une durée de $50 \mathrm{mn}$ à $1 \mathrm{~h} 30$ complète celle du verbatim des écrits numérisés comptant au maximum à 2800 mots.

Circonscrite au département «d'Ille et Vilaine » l'enquête s'adresse au personnel infirmier $(\mathrm{n}=110)$ exerçant dans des établissements publics d'enseignement en milieu rural, urbain et périurbain. Trente $(n=30)$ infirmières se sont portées volontaires.

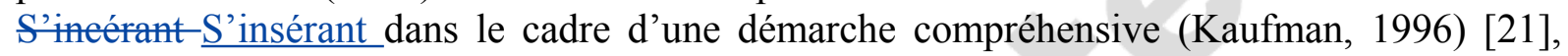
l'analyse herméneutique de ce corpus a pris la forme d'un découpage d'énoncés se référant à des « rubriques significatives » et pertinentes (Bardin, 2007) [25] quantifiées et répertoriées, ainsi qu'au repérage de formulations sémantiques suffisamment explicites.

\section{Résultats}

Les témoignages recueillis sous deux modalités associent les contenus de récits d'expérience $(n=13)$ à ceux délivrés sous la forme d'un entretien $(n=17)$. Scindé en deux parties ce panel distingue les infirmières exerçant sur plusieurs établissements (secteur + collège) à celles qui occupent des postes fixes de type lycée ou établissement régional d'enseignement adapté (Erea). Sous ces deux modalités de recueil, le corpus rassemble les énoncés issus des entretiens ( $n=719$ - acronyme « Et ») à ceux relevés dans les écrits ( $n=501$ - acronyme « $E c »)$. Les indicateurs conceptuels et opératoires choisis à la lumière de différents apports théoriques étayent les questionnements relatifs au développement professionnel dans ses dimensions à la fois développementales et professionnalisantes.

Produit d'interdépendances, le développement au travail articule le vécu personnel et professionnel ainsi que les facteurs environnementaux et institutionnels. L'évolution des pratiques et des modes de pensée imposent de prendre en considération les éléments relatifs aux conditions sociales, relationnelles et organisationnelles de l'exercice professionnel (Lefeuvre, Garcia \& Namolovan, 2009) [26]

Reflet d'une socialisation, le développement professionnel conjugue à la fois des relations, des modalités collectives d'organisation et de collaboration en lien aux spécificités structurelles de l'environnement (Martineau, Portelance \& Presseau, 2009) [27]. Les activités déterminent conjointement des ressources ou des contraintes qui favorisent ou inhibent selon le cas le potentiel développemental.

D'abord étudiée d'un point de vu développemental, l'évolution de carrière de l'infirmière détermine une entrée dans ce service en moyenne 10 ans après l'obtention du diplôme d'état (DE).

\footnotetext{
${ }^{1}$ Mots clés : rôle propre /expérience professionnelle/éducation/promotion de la santé/collectif (échanges, partages, appartenance)/doutes/sens/dilemmes/identité/reconnaissance/ projets/évolution.
} 
Dans une perspective professionnalisante le développement analyse le rôle propre sur la base des indicateurs théoriques de la posture largement évoquée dans cette enquête, des tensions identitaires citées en deuxième position, suivis des dimensions collectives de l'activité peu renseignées sur ces deux modalités de recueil (tableau 1.). Malgré le construit subjectif de ces catégories cette grille d'analyse permet de dégager des tendances significatives. L'expérience professionnelle s'identifie sans ambigüité d'abord à la posture (Et. $51 \%$ \& Ec. $52 \%$ ), et en particulier au travers du sens et des dilemmes que celle-ci soulève $(21 \%)$. Les dimensions identitaires citées en second lieu (Et. 32\% \& Ec. 35\%), sont suivies dans les entretiens par la place repérée du collectif dans l'exercice de la profession (Et.17 \% \& Ec.13\%).

Les infirmières ne font pas toujours la distinction entre la spécificité de ce rôle infirmier et leur posture «Je crois que c'est un tout, ce n'est pas dû qu'à la fonction çà n'appartient qu'à moi »; « Le rôle propre tu l'apprends sur le tas (...) j'ai jamais vraiment su ce que c'était que le rôle propre (...) « J'ai un mode de fonctionnement qui m'est très personnel, mais je pense que chacun dans ce métier développe la posture qui lui est propre »; «le rôle propre c'est ta définition personnelle du métier ». Dans sa dimension la plus explicite, le rôle propre dit aussi espace de «pratiques autonomes » est apprécié à l'aune des degrés de liberté qu'il offre dans l'organisation de l'activité ; « le travail est assez autonome et il permet de s'organiser comme bon nous semble ». Paradoxalement, les extraits codés dans la catégorie du sens et des dilemmes liés à cette posture sont largement dominants (24\% pour les Ec et $21 \%$ pour les Et). Le contenu de ce rôle reste difficile à expliciter et à appréhender pour un certain nombre d'entre elles. " Il m'est arrivé de me perdre dans cette posture, ce problème de posture ne s'est jamais posé à moi lorsque j'étais à l'hôpital, on est centré sur les besoins précis et prescrits des malades »; "Ce n'est pas facile de changer de posture surtout quand aucune formation sérieuse ne permet de réaliser ce grand écart (...) La profession d'acteur de prévention n'est pas celle de soignant, il a fallu endosser un autre costume (...) apprendre un nouveau job et enterrer le premier »; " il faut pouvoir exprimer toute l'ambiguité entre une formation de base dite hospitalière et le cadre professionnel de l'infirmière en $\operatorname{EPLE}_{-}^{2}(\ldots) \mathrm{J}$ 'ai toujours eu l'impression de ne pas devoir interroger ma posture à l'hôpital, ni même sa légitimité, c'était naturel $(. .$.$) ; « il faut apprendre à travailler seul (...) faire le deuil d'une posture strictement$ curative ». A l'interface des missions de soins, de relation d'aide et d'éducation à la santé les professionnelles interrogent la spécificité de leur expertise. "La légitimité et le sentiment d'utilité sociale dans le contexte est important. Il faut gagner du professionnalisme, mais ce n'est pas à travers l'éducation à la santé qui n'est pas une tâche très valorisée (...) Au début j'avais l'impression de faire un métier de maman (...) moi j'ai du mal à dire quelle est la spécificité de mon métier (...) c'est vrai le fait d'être une maman ce n'est pas très professionnel, çà fait penser au 'care' »; « J'aime les soins et c'est aussi ce qui me rassure il fait partie de ma fonction, le travail subjectif qui n'est pas rationalisable et qui n'est pas reconnu, on s'y perd (...) le rôle sanitaire est le premier reconnu (...) l'institution te pose une étiquette sur ton rôle de pompier, mais la voix du référent santé n'est pas souvent entendue (...) ».

Adossées aux missions d'éducation et de promotion de la santé les surveillances biométriques et l'aspect quantifiable de leur activité s'opposent à l'approche globale qu'elles ont de la santé à l'école. Ces bilans chiffrés leur semblent inadaptés voire ennuyeux. Elles questionnent cette logique de rationalisation de leur activité difficile à comprendre dans ces métiers de l'humain. «Par rapport aux grandes sections, on nous demande des choses qui ne sont pas réalisables, ces pressions imposent de faire du chiffre (...) on se demande le sens que ça peut avoir, mais l'institution aime le chiffre, dire que l'on a vu tant d'élève, tant de dépistage de trouble du langage "; " Les bilans à la chaîne ça vraiment je trouve ça inutile et inintéressant »; " (..) on m'a mise à faire des bilans, peser, mesurer, compter les dents, moi je n'ai jamais compris

${ }^{2}$ Etablissement public local d'enseignement 
ce que ça pouvait leur apporter aux petits (...) Et puis ça sert à quoi de mesurer une classe avec des gamins qui de toute façon finiront par grandir » (Et13).

De manière assez homogène la dimension identitaire de l'expérience professionnelle mobilise (32\% des Et \& $35 \%$ des Ec) des extraits codés. Ils désignent conjointement les tensions, mutations et transformations identitaires que suscite l'exercice en milieu scolaire. L'identité pour autrui reste liée aux représentations habituelles de la santé et de la maladie et se réfère aux acquis professionnels que leur a légués leur formation. Ces représentations résistantes font naître des doutes et desservent leur posture de référent santé que les infirmières ont de la peine à faire entendre. Remises en question dans le champ de la promotion de la santé les compétences sont perçues comme incertaines ; "Avant nous c'était des secouristes lingères et la représentation que l'on a de notre métier est toujours liée en partie à ce passé »; "Ton identité de soignante (...) c'est une gymnastique de tous les jours, c'est compliqué »; "il est difficile de faire face aux représentations collectives (...) de ce métier ». Vécu en termes de métamorphose, le remaniement identitaire touche à la fois les dimensions professionnelles et personnelles. À l'origine de doutes et de remises en causes, ces tensions identitaires sont variables selon les lieux d'exercice et l'entourage que partagent les autrui significatifs.

Les phénomènes identitaires analysés en termes de reconnaissance et positionnement représentent respectivement $(6 \%$ Et \& $7 \%$ Ec) des extraits codés. Ils concernent les attentes se référant aux attributions de l'activité et à ce qu'en attendent les infirmières elles-mêmes, en tant que sujet.

Les tensions identitaires (7\% des extraits codés des Et \& 14\% des Ec) s'appuient sur la remise en cause des valeurs professionnelles. Parfois teintées d'une perte d'estime de soi, le ressenti éclaire la difficulté de ne pouvoir qualifier ce travail humain toujours éprouvant. Le nonrespect du secret médical est perçu comme une atteinte aux valeurs professionnelles. Un soupçon semble peser sur leur sentiment de compétences et la reconnaissance du rôle spécifiquement infirmier.

Peu habituées au travail collectif et isolées de leurs collègues du fait de la configuration des postes, les infirmières identifient avec difficulté leur collectif professionnel d'appartenance. Cette rubrique a été la moins renseignée de toutes et représente pour l'ensemble $17 \%$ (Et) et $13 \%$ (Ec) des extraits codés. Partageant la double appartenance d'une équipe éducative et d'un collectif infirmier, elles questionnent l'objectif et la pertinence des échanges collectifs professionnels.

Les éléments les plus significatifs résumés ci-dessous en quelques points questionnent les limites que rencontre le développement du rôle propre, ainsi que les tensions et dilemmes relevés dans ce champ d'activité. 
Tableau 1.

Récapitulatif des codages réalisés sur les contenus : entretiens (Et) et écrits (Ec)

\begin{tabular}{|c|c|c|c|c|c|c|}
\hline & \multicolumn{3}{|c|}{ Contenus entretiens } & \multicolumn{3}{|c|}{ Contenus écrits réflexifs } \\
\hline \multirow{4}{*}{$\begin{array}{l}\text { 1. Posture Et } \\
\text { - } \quad 369 \text { extraits codés } \\
\text { - } \quad 51 \% \\
\text { 1. Posture Ec } \\
\text { - } \quad 262 \text { extraits codés } \\
\text { - } \quad 52 \%\end{array}$} & & Rôle propre/croyances/valeurs & $14 \%$ & 82 & Rôle propre/croyances/valeurs & $16 \%$ \\
\hline & & Santé/éducation & $5 \%$ & 28 & Santé/éducation & $6 \%$ \\
\hline & & Santé/promotion & $11 \%$ & 32 & Santé/promotion & $6 \%$ \\
\hline & & Sens/dilemmes/éthique & $21 \%$ & 121 & Sens/dilemmes /éthique & $24 \%$ \\
\hline \multirow{6}{*}{$\begin{array}{l}\text { 2. Construction identitaire Et } \\
-\quad 230 \text { extraits codés } \\
\text { - } \quad 32 \% \\
\text { 2. Construction identitaire Ec } \\
\text { - } \quad 170 \text { extraits codés } \\
\text { - } \quad 35 \%\end{array}$} & 35 & Dynamique/tension /identitaire & $5 \%$ & 17 & Dynamique/tension /identitaire & $4 \%$ \\
\hline & & Mutations transformation & $5 \%$ & 14 & Mutations transformation & $3 \%$ \\
\hline & 44 & Reconnaissance & $6 \%$ & 35 & Reconnaissance & $7 \%$ \\
\hline & & Dissonances/doutes/remises en causes & $7 \%$ & 68 & Dissonances, doutes, remises en causes & $14 \%$ \\
\hline & & Se positionner & $3 \%$ & 16 & Se positionner & $3 \%$ \\
\hline & 45 & Evolution projet & $6 \%$ & 20 & Evolution projet & $4 \%$ \\
\hline \multirow{3}{*}{$\begin{array}{l}\text { 3. Dimension collective Et } \\
\text { - } 120 \text { extraits codés } \\
\text { - } \quad 17 \% \\
\text { 3. Dimension collective Ec } \\
\text { - } 68 \text { extraits codés } \\
\text { - } \quad 13 \%\end{array}$} & 36 & Appartenance à un corps professionnel & $5 \%$ & 17 & Appartenance à un corps professionnel & $3 \%$ \\
\hline & & Compétences, genre /acculturation & $5 \%$ & & Compétences/genre /acculturation & $3 \%$ \\
\hline & & Échanges professionnels & $7 \%$ & 36 & Échanges professionnels & $7 \%$ \\
\hline
\end{tabular}

\section{Discussion : Les indicateurs de développement professionnel}

Quatre enseignements viennent nourrir cette recherche. Le premier souligne que le rôle propre est souvent synonyme de posture ou de "propre rôle». Définie comme un ensemble dynamique de croyances, d'intentions et d'actions, la posture guide l'activité (Lameul, 2006) [15].

La faiblesse du cadrage relatif aux activités de types discrétionnaires et l'incertitude des prescriptions qui les définissent laissent aux infirmier-ères une pluralité de modes opératoires. Pour atteindre leurs objectifs, elles improvisent des manières de penser et de faire, les conduisant à inventer une posture en lien aux formes nouvelles du milieu qui s'impose à elles. Difficile à définir chacun l'invente en fonction du contexte, du lieu d'exercice de sa personnalité, de ses valeurs et de ses représentations (l'infirmière d'une zone d'éducation prioritaire ne fait à priori pas le même travail que sa collègue exerçant en centre ville ou en zone périurbaine). Selon les publics qu'elle côtoie l'infirmière adapte la stratégie de ses interventions aux problématiques repérés dans les établissements, certains accueillent des élèves issus de milieux dits favorisés, lorsque d'autres organisent la scolarité de jeunes primoarrivants ne maitrisant pas toujours l'usage de la langue française (dans cette enquête des lycées et collèges recensent pour un même établissement la présence de plus de trente nationalités différentes).

Dans l'ensemble les caractéristiques de ce rôle ont été définies en termes de médiation, d'accompagnement, d'orientation et de relation d'aide. Dans cette fonction les soignants découvrent sans la nommer la dimension psychosociale « d'intermédiaire culturelle » orientée vers le service rendu aux élèves, à l'institution et aux familles, là où le soin n'a plus la même reconnaissance (Nadot, 2002,2005) [28] [29] ; Rothier-Bautzer, 2012, 2013) [3].

L'objectivation des pratiques relevant de la promotion de la santé est difficile à formuler et questionne la notion afférente de savoir infirmier. Face à l'incertitude des missions de prévention sous-traitées aux associations, les soignants éducateurs tentent pour s'y 
retrouver de se faire une place dans « la guerre » menée aux facteurs de risque et poursuivent la visée normative des dispositifs institutionnels tout en questionnant le sens et la cohérence de ces pratiques de prévention ponctuelles. S'ils sont justifiables sur la base des données épidémiologiques rien ne dit que l'empilement de ces actions soit compatible avec le rôle de l'école (Jourdan, 2007) [33].

$\mathrm{Au}$ centre d'un flou conceptuel et épistémologique associant les perspectives d'une éducation «à » la santé et d'une éducation "pour» la santé , les soignants éducateurs sont pris en tension entre les injonctions d'une éducation sanitaire issue des impératifs de santé publique ciblant prioritairement la prévention des consommations de produits psychoactifs et le déploiement d'une éducation « en » santé (Klein, 2010) [35], s'enracinant dans une définition plus holiste de L'OMS qui encourage aujourd'hui le développement des compétences psychosociales axées sur le travail de l'estime et la confiance en soi .

Le second point souligne les doutes émergents du consensus établi autour des pratiques sociales dites d'éducation à la santé renouant avec les origines sanitaires d'un paradigme biomédical qui vise l'amélioration des comportements (Klein, 2010) [35]. Issues des données épidémiologiques, le thème récurrent des consommations de produits psychoactifs, reste en tête de liste des interventions préconisées dans le département, mais il semble exclure la compréhension et l'origine tant psychosociale qu'environnementale des comportements de santé (Lecorps, 2012) [30]. Sceptiques, les infirmier-ères constatent l'inflation de « dys » et questionnent le choix des orientations sanitaires. En effet, celles-ci semblent réduire la complexité des problématiques de santé s'enracinant dans la mouvance des mutations sociales d'où émergent la violence, les cybers comportements, les reconfigurations familiales, l'absentéisme et le mal- être.

Les professionnels interrogent la pertinence et la définition des priorités classiques centrées sur les consommations d'alcool et autres produits psychotropes ainsi que la place réelle d'une éducation à la santé non inscrite dans le curriculum des élèves. Ces lacunes suscitent des questionnements lorsque ces actions sont laissées à l'initiative des infirmières elles-mêmes ou sous la tutelle d'un comité d'éducation à la santé et à la citoyenneté (CESC) dont le fonctionnement semble pour le moins disparate d'un établissement à l'autre.

Souvent restreinte à une information visant l'instruction, la santé enseignée sous la modalité d'une transmission ou d'un savoir de type biomédical semble insuffisante à l'orientation de comportements favorables. Pourtant, mises en évidence et relayées dans la littérature, les limites de ces approches pointent aujourd'hui l'organisation, l'efficience et les dimensions éthiques de ces actions dites d'éducation à la santé ; (Eymard, 2004) [32] ; (Jourdan, 2005) ; [32] (Berger, 2010) [32]; (Klein, 2010) [34]; (Guiet-Sylvain, 2011) [35]; (Gaussel, 2012) [36].

De nombreuses questions sont soulevées sur l'ambiguïté du terme même de promotion de la santé que les infirmières ont du mal à définir et qu'elles comparent souvent à un slogan. Ce dernier leur semble par ailleurs dissonant lorsqu'elles constatent elles-mêmes que l'école charrie son lot de violences symboliques (humiliation, harcèlement, stigmatisation, performance... ) et peut aller aussi à l'encontre des valeurs qu'elle prétend défendre. (Jourdan ....

La spécialisation du métier de soignant éducateur n'existe pas aux yeux des acteurs du terrain et les infirmier-ères en font tous les jours l'expérience se demandant même dans quelle mesure il peut être considéré comme un « cœur de métier ». Sans définition réelle il semble en effet problématique d'identifier la spécificité d'un rôle reposant sur l'entité d'un champ multidisciplinaire (Bury, 2001) [37] ; (Tuleu, 2001) [38].

$\mathrm{Au}$ centre d'un vieux débat qui oppose l'individu et son environnement les caractéristiques systémiques des déterminants de la promotion de la santé divisent. Fondée sur les postulats 
d'éducabilité, la démarche préventive est toujours celle d'une approche sociale et sanitaire inscrite dans la mouvance des problématiques culturelles, économiques et politiques. Mais objet par définition instable et vulgaire elle semble être l'espace de ces mondes, où se rencontrent sans dialoguer : la santé et l'éducation (Klein, 2010) [34]. Là où la bonne manière de faire reste légitimée et dictée par des experts de l'OMS (Lecorps, Paturet, 1999) [5], les paradigmes s'observent sans se croiser (Fortin, 2004) [39] et la réification de l'activité soignante repose pour l'essentiel sur un modèle de conformation.

La professionnalisation pour le coup inaboutie dans ce champ de la prévention doit prendre en considération les apports de la recherche et les résultats insatisfaisants des actions de prévention (Guiet-Silvain, 2011) [35] ; Gaussel, 2012) [36]. C'est en réinterrogeant la construction des référentiels de compétences, les pratiques formatives et les rhétoriques professionnelles que peut être redéfinie la place des sciences humaines et sociales dans l'appréhension et la gestion des phénomènes de santé (Jourdan, 2004) [32] ; (Dominicé, Jacquemet, 2009) [11].

Le rôle propre se heurte aux contradictions d'une activité dictée par les logiques organisationnelles «du devoir-agir» (Triby, 2012) [40]. Dominées par des approches pragmatiques et fondées sur un effort de rationalisation supposé offrir le sentiment de maîtrise du réel, ces prescriptions entrent en conflit avec les valeurs professionnelles nécessitant d'interroger l'activité et le développement professionnel des acteurs qui en ont la charge.

Amputée de sa dimension réflexive, éthique et critique la professionnalité infirmière reste aliénée aux dispositifs ne visant que les résultats d'une activité comptable et performante du service public. Les pratiques de quantifications, chronophages et largement controversées dénaturent et vident le sens donné au travail (Gaulejac (de), 2005) [41] ; (Abelhauser, Gori, Sauret, 2011) [42].

$\mathrm{Au}$ centre d'une reconfiguration professionnelle qui manque de cohérence, le soignantéducateur opte alors pour le consensus et élimine les scories de l'affect préférant l'aseptisation d'un rapport pragmatique au métier. S'opposant aux valeurs d'une prise en charge globale de l'individu, ces pratiques prescrites de santé publique ne signent en rien le déploiement d'un raisonnement infirmier devenu incertain. Difficile à apprécier, la valeur de ce rôle propre est complexe et ne rentre pas dans les cases habituelles de ces évaluations. Condamnée à l'invisibilité et au silence cette activité sous évaluée n'est pas valorisée et dans ce champ d'exercice, les infirmières interrogent la validité de leur expertise.

Le troisième point concerne la reconnaissance de ce rôle propre infirmier au niveau de la société en général et de l'institution en particulier. Quel crédit accorde t'on au savoir infirmier? D'abord perçue comme une auxiliaire médicale, la place de l'infirmière reste liée au mandat social qui la définit. Son champ d'activité vise principalement le soin des maladies et la réduction des facteurs de risque catégorisés sur le modèle biomédical de la pathogenèse. Mais la lente évolution des modèles et des représentations continue d'alimenter les clichés d'une profession toujours dépendante des savoirs médicaux. L'absence de consensus autour de la définition même de la santé, génère une grande variété de représentations abandonnées souvent aux idéologies dominantes (Descarpentries, 2008) [6] ; (Klein, 2010) [34]. De ce fait la voix du référent santé reste difficile à faire entendre et mal identifiée par les professionnelles elles-mêmes. Or les infirmières de la promotion de la santé souhaiteraient faire évoluer les représentations de secouristes. Diffus le discours de la « salutogenèse » n'est pas entendu et difficile à mettre en place. La notion même de promotion de la santé reste vague et nébuleuse aux regards des professionnels qui constatent sur le terrain que l'éthique d'une promotion de la santé est parfois bien éloignée des promesses du «care 》 (Molinier, 2006) [43]; (Paperman, 2013) [44]. 
Enfin dans un dernier point et à l'instar des précédentes enquêtes, l'incertitude professionnelle exposée aux caractéristiques de l'isolement domine. Privées d'analyse de pratique, les infirmier-ères soulignent la faiblesse des ressources collectives auxquelles s'ajoutent les lacunes d'une formation continue jugée redondante et souvent inadaptée à leur besoin (OsiekParisod, 1994) [17]; (Batt, 1999) [18] ; (Berger, 2009) [8].

\section{Poursuivre la recherche}

Si la recherche a tenté ici d'appréhender l'analyse du rôle propre à l'aune du développement professionnel, elle nous indique que les infirmières identifient avec difficulté le contenu de ce rôle afférent au concept de promotion de la santé.

Pourtant symbole d'une émancipation, ce rôle propre repose sur un paradoxe. Bien que la professionnalisation du soignant éducateur suive le programme institutionnel de la Santé Publique, la référence aux soins infirmiers n'est pas sans ambiguïtés et source de questionnements chez les professionnels contraints d'associer les approches individuelles et collectives (Pommier, Deschamps, 2004) [45]; (Jourdan, 2004, 2007) [19] [32].

Cette étude souligne aussi que les missions des professionnels de santé scolaire centrées sur des préoccupations liées à l'hygiène, à la sécurité et aux soins se sont déplacées vers des préoccupations sociales nouvelles (Sandrin-Berthon, 2006) [46]; (Lecorps, 2012) [30].

Inaccompli, le rôle propre a bien des défis à relever et le statut de professionnelle de santé reste encore à construire. Si l'attribution de ce rôle marque l'avènement d'une nouvelle discipline professionnelle de la santé, alors quel bilan est-il possible de dresser 40ans après ? Quelle est la nature des savoirs infirmiers ? En dépit des initiatives destinées à favoriser la construction identitaire et l'évolution des représentations de la fonction infirmière, le mandat social qui les définit dans ce champ de la promotion de la santé reste vague. L'organisation et la structure des soins complexifient la définition préventive du rôle infirmier et conforment les représentations de la population à l'égard des infirmières dont l'activité reste sous-évaluée. Les valeurs biomédicales restent dominantes et le manque d'unité professionnelle complique ce processus (Debout, 2008) [47].

La professionnalisation des soignants éducateurs interroge directement la définition de leurs compétences et demande de prendre en compte les recherches dans le champ de sciences humaines et sociales (Jourdan, 2004) [19]; (Dominicé, Jacquemet, 2009) [11]. Symptomatiques d'une logique qui dicte des normes, les dispositifs de prévention sont mis en place dans le déni de l'activité réelle. Eminemment clinique, la santé scolaire reste bien éloignée des énoncés épidémiologiques et les infirmières questionnent sur le terrain la pertinence des taxinomies imposées lorsqu'elles observent que les problèmes s'enracinent souvent ailleurs.

Pour définir la singularité de ce savoir qui circule et s'hybride sur la réalité des terrains, la démarche imposera sans doute de sortir de la seule problématique de compétence qui définit ce rôle propre insuffisant et peu fécond pour s'intéresser à la spécificité des soins infirmiers, à leur objet et aux connaissances qui s'y rapportent. Car la notion de rôle propre sous-tend en effet celle d'une pensée propre (Poisson, 2008) [50].

Aujourd'hui le processus de professionnalisation impose le recours à la formalisation de l'expérience et présuppose que les infirmières soient en mesure d'expliciter et de justifier de leurs actes par des analyses orales et écrites (Debout, 2008) [47]. Seule cette posture réflexive peut leur permettre de se distancier de la raison pratique (Nadot, 2002) [27] ; (Jovic, 2008) [48]; (Rothier-Bautzer, 2012) [3]. Mais peu habituées à objectiver leur activité sur le terrain, elles éprouvent des difficultés à décrire et à parler de leur rôle propre. Contraintes depuis leurs études à adopter la forme d'écrits standardisés, les infirmier-ères se plient aux injonctions administratives réduisant de plus en plus leur autonomie scripturale. Conditionnés, les 
soignants renseignent les menus condensés de logiciels destinés à la quantification des logiques gestionnaires. Objets de pratiques protocolaires et objectivantes, le prendre soin devient la pensée improbable d'un environnement qui se médicalise à contre-courant d'un discours holiste et humaniste au sein d'une culture où il n'est plus considéré comme une valeur (Perrault-Soliveres, 2001) [49] ; (Molinier, 2006) [43] ; (Paperman, 2013) [44].

\section{Conclusion}

Au centre du rôle propre, le postulat du savoir infirmier repose sur une conception globale se référant au prendre soin. Objet d'une praxéologie axée sur l'analyse des processus de vie (Collière, 1982) [1], il sollicite différents savoirs au cœur d'une activité pluridisciplinaire, (apparaissant «à la fine pointe » des sciences humaines et sociales (Nadot, 2002, 2005) [27] [28].

Mais peut-être est-il temps, en effet de dépoussiérer ce rôle. Si les infirmières ont à cœur de promouvoir la vie autant que la santé (Collière, 1982) [1], sans doute faudra-t-il pousser l'analyse critique et opposer un véritable raisonnement sur la base d'un jugement axiologique et scientifiquement fondé afin qu'il puisse trouver la voix d'un argument professionnel légitime (Debout, 2008) [47] ; (Jovic, 2008) [48]; (Pacific, 2013) [52].

Quelle posture professionnelle et épistémique convient-il d'adopter pour favoriser cette émergence ? Quelle nécessité éthique, aux confluents des métiers du soin et de la relation? Favorisera t-elle une pensée distanciée et critique des professionnels sur leurs trajectoires ?

La posture s'enracine sans doute dans notre capacité de dissidence et de différence, mais la pensée autonome nécessite de bien définir la promotion de la santé en lien aux pratiques dites du «care ». Si le sous-bassement du prendre soin semble être une vieille distinction entre la raison et les sentiments, il ne peut déprécier le savoir pour privilégier une sensibilité et un humanisme souvent inconsistants dont on ne sait pas trop ce qu'ils sont. Mieux comprendre rationnellement "c'est mieux sentir et mieux faire » (Perrier, 2012, 141) [52]. Le "care » doit aussi soumettre l'expérience à l'effort de sa compréhension rationnelle, pour devenir plus juste, mieux sensible, plus efficace et moralement source d'une bien plus grande compétence. Même s'il existe toujours des conflits épistémologiques entre les sciences de la santé et les sciences humaines (Descarpentries, 2006) [10]; (Dominicé, Jacquemet, 2009) [11], les soignants peuvent orienter la connaissance et prendre la parole pour ne pas la laisser aux privilèges du discours technocratique des chefs de projets souvent à l'écart des réalités. Dans la continuité des travaux de Perraut Solivérès (2001), la posture de praticien-chercheur revendiquée ici, offre des manières renouvelées d'observer les routines professionnelles qui ne sont plus questionnées.

La professionnalisation est à poursuivre car les infirmier-ères ont aujourd'hui le défi de s'approprier ce rôle autonome et la fonction indépendante de leur profession centrée sur la promotion de la santé (Piguet, 2008) [2]. Bien que l'universitarisation et les réformes successives tentent de promouvoir un nouveau modèle, l'unique façon de s'en sortir sera de démontrer que ces compétences existent et qu'elles constituent une plus-value. Mais seules les exigences de l'écriture permettront de transformer l'expérience en savoir scientifiquement valide. C'est pour cela que la recherche en soins infirmiers est une opportunité et une voie vers la reconnaissance.... et ça, c'est plutôt une bonne nouvelle. 


\section{Remerciements}

Cette étude a été réalisée avec le soutien académique de Jérôme Eneau et de Marc Nagels

Université européenne de Bretagne

Département des Sciences de l'Education

Place du Recteur Le Moal.

35000 Rennes

\section{Références bibliographiques}

1. COLLIERE MF. Promouvoir la vie. De la pratique des femmes soignantes aux soins infirmiers. Paris, Interedition, 1982.

2. PIGUET C. Autonomie dans les pratiques infirmières hospitalières : contribution à une théorie agentique du développement professionnel. Thèse de doctorat en sciences de l'éducation et santé publique. Sous la co-tutelle de l'Université Catholique de Louvain et l'Université Paris X- Nanterre. 2008.

3. ROTHIER-BAUTZER E. Entre le cure et le care. Les enjeux de la professionnalisation infirmière. Paris, Éditions Lamarre, 2012.

4. PEPIN J, KEROUAC S, DUCHARME F. La recherche au service de la pratique. In La pensée infirmière. Chenelière Edition, 2010.

5. LECORPS P, PATURET JB. Santé publique du biopouvoir à la démocratie. Paris, ENSP, 1999.

6. DESCARPENTRIES J. Essai de Conceptualisation de l'intervention éducative en santé publique. Promotion \& Education $\mathrm{n}^{\circ} 1$, 14-16. 2008. En ligne : http://ped.sagepub.com/content/15/1_suppl/14.full.pdf consulté le 8 décembre 2011 .

7. THOMANN T, GABEL M, SENETERRE E. L'infirmière scolaire aujourd'hui. Paris, Maloine, 1998.

8. BERGER D, MABROUCK N, COURTY P. Infirmiers scolaires : représentations et pratiques d'éducation à la santé. Santé publique $2009 ; 6: 641-657$.

9. NAGELS M. Construire son auto-efficacité. Construire ses compétences. 2013. En ligne :http://www.17marsconseil.fr/blog/2013/10/29/construire-son-auto-efficaciteconstruire-ses-competences/, consulté le 10 juillet 2013.

10. DESCARPENTRIES J. Introduction. Les Sciences de l'éducation- pour l'Ère nouvelle. 2006. En ligne : 39,7-15.http://.cairn.info/revue-les-sciences-de-l-educationpour-1-ere-nouvelle-2006-1-page-7. htm consulté le 12 décembre 2012. 
11. DOMINICE P, JAQUEMET S. Formation et Santé. Savoirs, 19, 7-36. 2009. En ligne : http://www.cairn.info/revue-savoirs-2009-1-page-7.htm consulté le 24 novembre 2012.

12. LE MOIGNE JL. Les épistémologies constructivistes. Paris, P U F, 2007.

13. WITTORSKI R. Professionnalisation et développement professionnel. Paris, L'harmattan, 2007.

14. RABARDEL P. Instrument subjectif et développement du pouvoir d'agir. In : P. Rabardel \& P. Pastré (Ed.). Modèles du sujet pour la conception. Dialectiques, activités, développement .Toulouse, Octarès, $2005: 11-29$.

15. LAMEUL G. Former des enseignants à distance? Etude des effets de la médiatisation de la relation pédagogique sur la construction des postures professionnelles, thèse de doctorat en sciences de l'éducation. Université de Paris-X-Nanterre. 2006.

16. WITTORSKI R. ARDOUIN T. La professionnalisation : étudier la complexité des liens sujet-organisation. In : CLENET J, MAUBANT PH, POISSON P. Formations et Professionnalisations : à l'épreuve de la complexité. Paris, l'Harmattan ,2012 : 91-106.

17. OSIEK-PARISOD F. Infirmière scolaire, une place à trouver et à faire reconnaître. Objectifs Soins, $1994 ; 25: 46-50$.

18. BATT A. Santé ressentie et implication au travail des infirmier-ères de l'éducation nationale. Santé publique, $1999 ; 4$ : (11), 493-501.

19. JOURDAN D. La formation des acteurs de l'éducation à la santé en milieu scolaire. Toulouse : Editions Universitaires du Sud, 2004.

20. MAYEN P. Quelques repères pour analyser les situations dans lesquelles le travail consiste à agir pour et avec un autre. Recherches en Education, revue du centre de recherche en éducation de Nantes, 4, 51-64. 2007. En ligne : http://www.crennantes.net/IMG/pdf/Revue REE no4.pdf consulté le 8 Novembre 2012.

21. KAUFMAN JC. L’Entretien compréhensif. Paris, Armand Colin, 1996.

22. CIFALI M, ANDRE A. Ecrire l'expérience. Vers la reconnaissance des pratiques professionnelles. Paris, PUF, 2007.

23. VINATIER I. Quel dispositif d'analyse pour permettre à des professionnels de terrain de s'approprier leur activité. In : VINATIER I (et al.). Réflexivité et développement professionnel. Une orientation pour la formation. Toulouse, Octares, 2012 : 38-57.

24. DUBET F. Rôle et expérience. In : L'analyse de la singularité de l'action. Centre de recherche sur la formation du Cnam. Education et Formation. Paris, PUF, 2000 ; $72-$ 83.

25. BARDIN L. L'analyse de contenu. Paris, PUF, 2007. 
26. MARTINEAU S, PORTELANCE L, PRESSEAU A. La socialisation au travail comme indicateur de développement professionnel : analyse des approches basées sur la mesure. Questions Vives Le développement professionnel : quels indicateurs ? Vol.5 $\mathrm{n}^{\circ} 11$. En ligne : http://questionsvives.revues.org/614 consulté le 22 février 13 sur

27. LEFEUVRE G, GARCIA A, NAMOLOVAN L. Le développement professionnel. Quels indicateurs? Questions vives. 2009 ; Vol.5 n¹1. En ligne : questionsvives-627vol-5-n-11-note-de-synthese-les-indicateurs-de-developpemeprofessionnel\%20(1).pdf consulté le 10 Décembre 2013.

28. NADOT M. Médiologie de la santé. De la tradition soignante à l'identité de la discipline. Perspective soignante, $\mathrm{N}^{\circ} 13$. Paris, Seli Arslan, 2002.

29. NADOT M. Au commencement était « le prendre soin ». Soins, $2005 ; 700: 37-40$.

30. LECORPS P. Les conditions « du vivre». La Santé de l'Homme, 2012; 420 : 56 Hors-série.

31. EYMARD C. Essai de modélisation des liens entre éducation et santé. Questions Vives $n^{\circ} 5$, Volume 2, Education à la santé ou pour la santé ? $2004 ; 5: 13-33$.

32. JOURDAN D. L'éducation à la santé à l'école. Apprendre à faire des choix libres et responsables. 2007. En ligne : http://www.cairn.info/article_p.php? ID_ARTICLE=SEVE_016_0053 consulté le 26 septembre 2011.

33. BERGER, D. Quelle(s) formation(s) en éducation à la santé. ". $3{ }^{\text {ème }}$ Colloque réseau des universités pour l'éducation à la santé. 2010. En ligne: http://platesformes.iufm.fr/education-santeprevention/IGM/pdf colloque UNIReS consulté le 4 octobre 2012.

34. KLEIN A. Education en santé au secours des sciences de l'éducation : prémisses à un essai d'épistémologie croisée. Actes du congrès de l'actualité de la recherche en éducation et en formation AREF 2010. En ligne : https://plone2.unige.ch/aref2010/.../Leducation\%20en\%20sante.pdf// consulté le 11 février 2011.

35. GUIET-SILVAIN J. Education à la santé en milieu scolaire, mise en perspective historique et internationale. Carrefours de l'éducation, $2011 ; 32: 105-127$.

36. GAUSSEL M. Vers une école saine, éducation à la santé, volet 2. Dossier d'actualité veille et analyse. Institut Français de 1'Education, $N^{\circ}$ 77. 2012.

37. BURY J. La professionnalisation en éducation et promotion de la santé : Oui mais... La santé de l'Homme $2001 ; 353: 57-59$.

38. TULEU F, Trajectoires personnelles et professionnelles. La santé de l'Homme, 2001 ; $353: 13-15$.

39. FORTIN J. La formation des acteurs de l'éducation à la santé en milieu scolaire. Toulouse, Editions Universitaires du Sud, 2004. 
40. TRIBY E. Le devoir-agir, un concept pour comprendre les obstacles à l'émancipation en santé et éducation. Colloque international Rennes, Formes d'éducation et processus d'émancipation en santé et éducation, 2012.

41. GAULEJAC (de), V. La société malade de la gestion. Paris, Le Seuil, 2005.

42. ABELHAUSER A, GORI R, SAURET, MJ. La Folie Evaluation. Les nouvelles fabriques de la servitude. Paris, Fayard, 2011.

43. MOLINIER P. "Le care à l'épreuve du travail. Vulnérabilités et savoir-faire discrets ", dans P.Paperman, S.laugier (dir.). In Le souci des autres. Paris, EHESS Editions, 2006.

44. PAPERMAN P. Care et sentiments. Paris, P U F, 2013.

45. POMMIER J.DESCHAMP JP. Les infirmières et la santé publique : Evolution des missions et des formations, Santé Publique $2004 ; 16$ : 197-206.

46. SANDRIN-BERTHON B. Promotion de la santé : De la théorie à la pratique .... Ou à quoi servent les déclarations de l'OMS ? Education Santé. 216, 1-5. 2006. En ligne : www.educationsante.be/es/article.php?id=824 consulté le 12 octobre 2011.

47. DEBOUT C. «Sciences des soins infirmiers : réflexions épistémologiques sur le projet d'une discipline », Recherche en soins infirmiers 2008 ; 93 : 72-82.

48. JOVIC L. Les soins infirmiers en France aujourd'hui : problèmes et perspectives. Les conditions d'émergence d'une discipline scientifique et professionnelle. Recherche en soins infirmiers, $2008 ; 93: 68-71$.

49. PERRAULT-SOLIVERES A. Infirmières, le savoir de la nuit. Paris : P U F, 2001.

50. POISSON M. Les soins infirmiers en France aujourd'hui : problèmes et perspectives. Le pansement et la pensée : splendeurs et misères du rôle propre. Recherche en Soins Infirmiers, 93, 56-60.

51. PACIFIC C. Le soin : recherche de consensus ou principe de conflit nécessaire? Recherche en soins infirmiers, $2013 ; 114: 14-20$.

52. PERRIER S. L'autonomie doit-elle être la finalité de l'éducation ? Portée et limites d'une critique care de l'autonomie. Eduquer la liberté, fragilité consubstantielle à l'identité humaine. Revue Transverse, 2012 ; 3 : 104-146. 


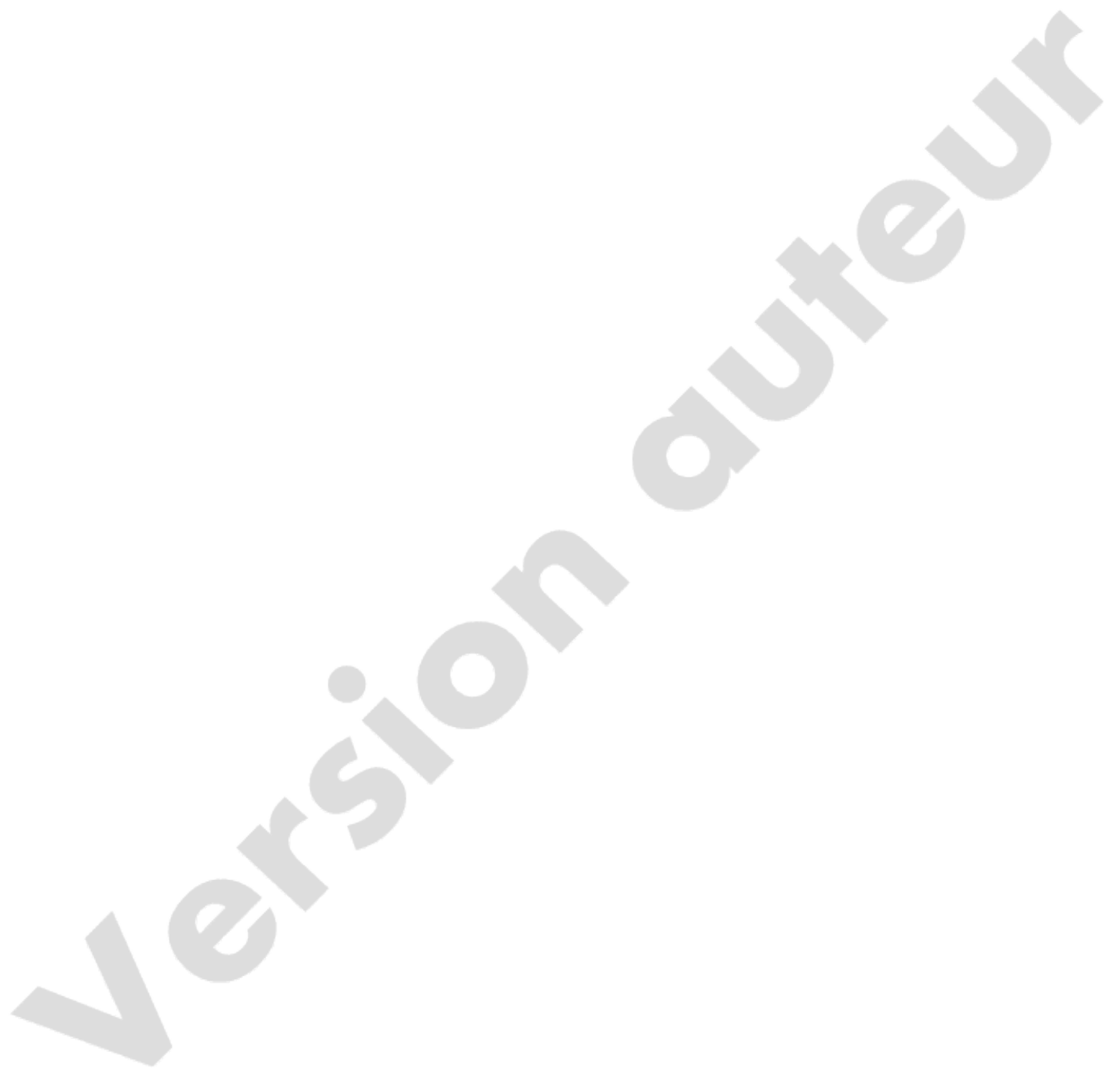

